\title{
Radiotherapy in the treatment of aggressive fibromatosis: experience from a single institution
}

\author{
K. Seidensaal $1^{1,2,3,4}$, S. B. Harrabi $i^{1,2,3,4,5^{*}}$ (D) F. Weykamp ${ }^{1,2,3,4}$, K. Herfarth ${ }^{1,2,3,4,5}$, T. Welzel ${ }^{1,2,3}$, G. Mechtersheimer ${ }^{6}$, \\ B. Lehner ${ }^{7}$, M. Schneider ${ }^{8}$, S. Fröhling ${ }^{9}$, G. Egerer ${ }^{10}$, J. Debus ${ }^{1,2,3,4,5,11}$ and M. Uhl ${ }^{1,2,3,4}$
}

\begin{abstract}
Background: Desmoid-type fibromatosis is a rare, potentially locally aggressive disease. Herein we present our experience in the treatment with radiotherapy.

Methods and materials: In total 40 patients who received 44 treatments from 2009 to 2018 at the Heidelberg University Hospital with photons $(N=28)$ as well as protons $(N=15)$ and carbon ions $(N=1)$ were investigated. The median age at radiotherapy was 41 years [range 8-78]. Familial adenomatous polyposis (FAP) was confirmed for nine patients and 30 had a unifocal desmoid tumor. The localizations were abdominal wall, abdominopelvic cavity, thoracic wall, extremity, head and neck and trunk. The median prescribed dose was 54 Gy/ Gy (RBE) [range 39.6-66, IQR 50-60]. Eleven treatments were performed at the time of first diagnosis; 33 at the time of progression or recurrence. Post-operative radiotherapy was performed in 17 cases. The median planning target volume was $967 \mathrm{ml}$ [84-4364 ml, IQR 447-1988]. Survival analysis was performed by the Kaplan-Meier Method.

Results: The median follow-up time was 32 months [1-153]. At the end of the follow-up interval all patients but one were alive. The estimated local progression free survival of the treated lesion in 3 and 5 years was $76.4 \%$ and $63,8 \%$, respectively. The progression-free survival in 3 and 5 years was 72.3 and $58.4 \%$ and the overall survival was 97.4 and $97.4 \%$, respectively. In case of macroscopic tumor $(N=31)$ before radiotherapy a partial remission was observed in 12 cases (38.7\%) and a complete remission in 4 cases (12.9\%). Progression was observed in 13 (29.5\%) cases, predominantly at the margin of the planning target volume (PTV, $N=5,38,4 \%$ ) followed by progression within the PTV $(N=4,30.8 \%)$. In univariate analysis multifocal localization was associated with impaired progressionfree survival $(p=0.013)$. One patient developed a grade $V$ gastrointestinal bleeding, otherwise no acute toxicity $>^{\circ} \| I$ was observed. Late toxicity was depending on the localization of the desmoid tumor and was especially severe in patients with FAP and abdominopelvine desmoids including gastrointesinal fistula, perforation and abscess.

Conclusion: Radiotherapy in the treatment of desmoids can lead to long term control. Treatment of patients with abdominopelvine desmoids should be avoided, as the risk of higher-grade complications is substantial.
\end{abstract}

Keywords: Aggressive fibromatosis, Desmoid tumor, Radiotherapy, Proton therapy

\footnotetext{
* Correspondence: semi.harrabi@med.uni-heidelberg.de

${ }^{1}$ Department of Radiation Oncology, Heidelberg University Hospital, Im Neuenheimer Feld 400, 69120 Heidelberg, Germany

${ }^{2}$ Heidelberg Institute of Radiation Oncology (HIRO), Heidelberg, Germany

Full list of author information is available at the end of the article
}

(C) The Author(s). 2020 Open Access This article is licensed under a Creative Commons Attribution 4.0 International License, which permits use, sharing, adaptation, distribution and reproduction in any medium or format, as long as you give appropriate credit to the original author(s) and the source, provide a link to the Creative Commons licence, and indicate if changes were made. The images or other third party material in this article are included in the article's Creative Commons licence, unless indicated otherwise in a credit line to the material. If material is not included in the article's Creative Commons licence and your intended use is not permitted by statutory regulation or exceeds the permitted use, you will need to obtain permission directly from the copyright holder. To view a copy of this licence, visit http://creativecommons.org/licenses/by/4.0/. The Creative Commons Public Domain Dedication waiver (http://creativecommons.org/publicdomain/zero/1.0/) applies to the data made available in this article, unless otherwise stated in a credit line to the data. 


\section{Introduction}

Aggressive fibromatosis describes rare benign lesions, which arise from musculoaponeurotic structures. The alternative term "desmoid" refers to the tendon like appearance. They account for $0.03 \%$ of new diagnosed neoplasms and for $3 \%$ of soft tissue tumors [1]. The treatment options include surgery, radiotherapy, hormonal therapy, chemotherapy and anti-inflammatory agents [2]. Despite their clinically circumscriptive appearance, histologically those tumors show diffuse and infiltrative growth into surrounding tissues and are not encapsulated [3]. The course of the disease is variable and unpredictable including growth, progression, stabilization, and even spontaneous regression [4]. Thus, for most patients an observation period is advisable [5]. When active therapy is necessary, surgery is one option. Wide microscopic margin-negative resection (R0) is the goal, but conservation of cosmesis and function is the major priority [6]. Despite the tendency for local recurrence, desmoid tumors do not metastasize but they can present multifocal in the same limb or region. Preceding traumatic events to the involved area, previous pregnancy as well as surgical scars have been identified as factors contributing to the development of aggressive fibromatosis. Desmoids occur sporadic but are also associated with familial neoplastic syndromes. Especially at the abdominal site desmoids are often encountered in patients with familial adenomatous polyposis coli (FAP, Gardners syndrom). Treatment of aggressive fibromatosis is complex and depending on the anatomical location and size of the tumor. Mortality might be low but the risk of significant disability and morbidity is crucial [7]. Herein we present our single center experience in the treatment of desmoid tumors by radiotherapy, the focus of the study is to assess i) treatment related morbidity, ii) patterns of response or treatment failure, and identify iii) opportunities to improve treatment strategies.

\section{Methods and materials Data collection}

The Ethics committee of the University of Heidelberg approved the study. Data on patients', tumor and treatment characteristics, imaging modalities/ results and outcome were obtained from retrospective review of medical records. The following variables with the according thresholds were analyzed for their prognostic value: gender, age at radiotherapy (median of 41y), FAP (yeas/no), tumor size described by PTV (median of 967 $\mathrm{ml}$ ), tumor site (abdominal vs. extraabdominal), timing of treatment (primary situation vs. recurrence/progression), macroscopic tumor at the time of treatment (yes/ no), applied dose (median of 54Gy/Gy (RBE) and threshold of 50Gy/Gy (RBE)), previous surgery (yes/no), previous medical treatment (yeas/no), particle vs. photon radiotherapy and definitive radiotherapy vs. postoperative radiotherapy. .

\section{Study cohort}

Forty consecutive patients who have been treated at the University Hospital Heidelberg August 2009-December 2018 were included in this study. One patient received a previous treatment at our intuition in April 2006 which was also considered. In total the patients received 44 courses of radiotherapy. Of the four patients treated twice, three patients developed new desmoids in proximity to the previous lesion (intraabdominal/ abdominal wall $N=2$, lower extremity $N=1$, example Fig. $1 \mathrm{~A}$ and $\mathrm{B})$, and one patient progressed at the cranial margin of the previous desmoid tumor. Five patients were excluded as no follow up was available, two previous courses of radiotherapy dated back longer were not included as the data was insufficient for analysis. The median age at first presentation was 41 years [8-78], 30 patients $(75 \%)$ had one single desmoid. The treated lesions were located in the abdominopelvic cavity $(N=11,27.5 \%)$, thoracic wall $(N=9,22.5 \%)$, extremities $(N=9,22.5 \%)$, abdominal wall $(N=4,10 \%)$, trunk $(N=4,10 \%)$ and head and neck $(N=3,7.5 \%)$. Nine patients had a familial adenomatous polyposis (FAP, Gardner's syndrome), the diagnosis desmoid tumor of five of that was based on morphological imaging and not histologically confirmed. Five FAP patients had previous surgery in the region of the desmoid tumor, thereof four a prophylactic proctocolectomy and one a gastrectomy (Table 1$)$.

The majority of treatments was performed not at the time of first diagnosis $(N=11,25 \%)$ but at the time of progression or recurrence $(N=33,75 \%)$. Twenty-seven treatments were performed by radiotherapy only and 17 as postoperative additive radiotherapy for microscopic (R1) or macroscopic (R2) incomplete resection. In 31 cases gross tumor was detectable on planning computed tomography.

Previous treatment included surgery and medical treatment and are summarized in Table 2. Only 14 (31.8\%) had no prior surgery, 18 (40.9\%) had one prior resection of the desmoid tumor, six (13.6\%) two prior resections and six (13.6\%) more than two prior resections. Seven treatments were performed as a second course of percutaneous radiotherapy, five had a clear overlap of the high dose area, one had no overlap and one was in a different region. Two patients with thoracic desmoids had previous radiotherapy to the chest for breast cancer.

In total 25 patients received no previous medical treatment, the rest of the cohort received various substances and combinations, including sulindac $(N=9)$, meloxicame $(N=2)$, celecoxib $(N=1)$, diclophenac $(N=1)$, tamoxifen $(N=7)$, raloxifene $(N=1)$, toremifen $(N=1)$, imatinib $(N=5)$, methotrexathe $(N=2)$, vinchristine 

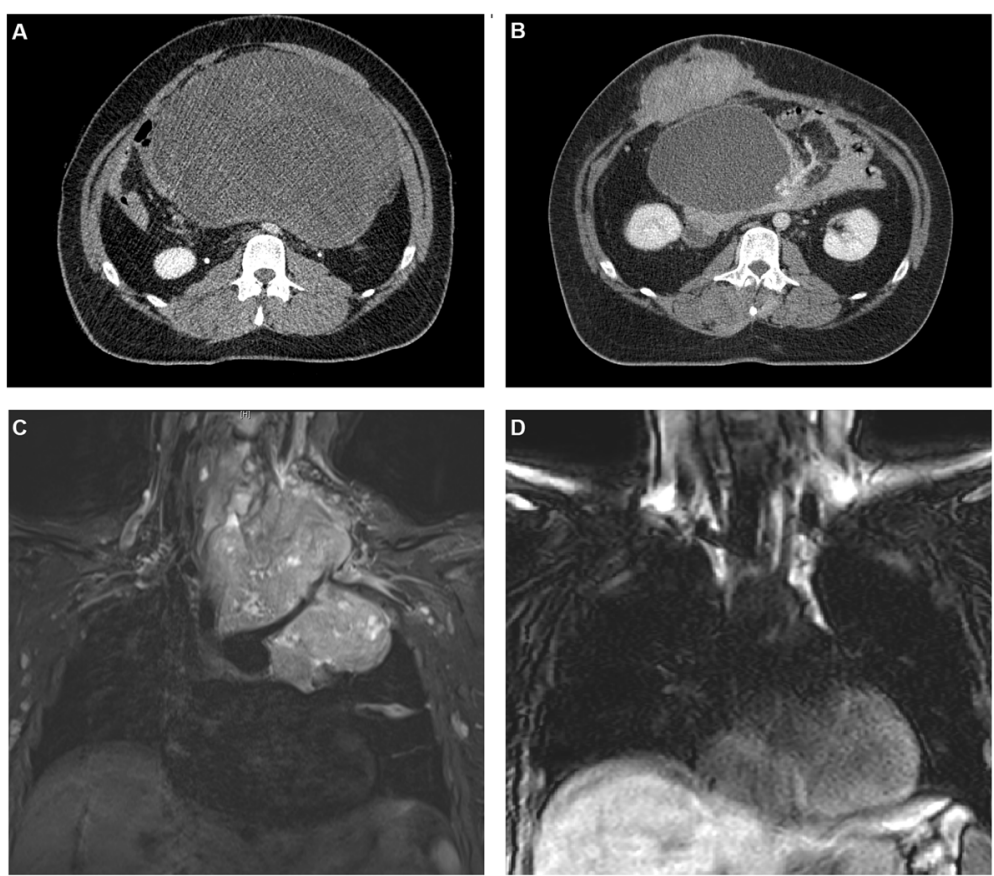

Fig. 1 Examples of patients with desmoid tumors: A 27-year-old patient with a progressive, gigantic, FAP-associated mesenteric desmoid tumor, treatment was performed with photon-IMRT up to 50.4 Gy in 28 fractions, B: The patient developed a new symptomatic desmoid tumor of the abdominal wall 7 years after the primary treatment and was treated with protons 56 Gy (RBE) in 28 fractions. The mesenteric desmoid regressed in size, and stabilized with a cystic appearance on CT and MRI (not shown). C: Thirty-two-year-old patient who was pregnant at diagnosis of a gigantic cervico-thoracic desmoid tumor. The patient was treated beginning in the 28th week of pregnancy with protons, however the tumor progressed rapidly and the treatment was discontinued at 30 Gy (RBE). Caesarean section was performed and treatment with methotrexate and vinorelbine initiated. The tumor regress significantly after that

$(N=2)$, doxorubicin $(\mathrm{N}=5)$, actinomycin $\mathrm{D}(N=1)$, cyclophosphamide $(N=1)$, dacarbazine $(N=3)$, One patient received a multiagent-chemotherapy, details on the substances were not available.

\section{Treatment planning and radiotherapy details}

Patients were treated at Heidelberg Ion-Beam Therapy Center (HIT) and the Heidelberg University Hospital. Treatment characteristics are summarized in Table 3. Treatment with protons and carbon ions was performed exclusively with active raster scanning as previously published $[8,9]$. Treatment with photons was performed as 3D conformal radiotherapy or intensity modulated radiotherapy (IMRT), either helical IMRT or IMRT in VMAT technique at the discretion of the according radiation oncologist. Intraoperative electron radiation therapy (IOERT) was performed by The Mobetron ${ }^{\circ}$ (IntraOP Medical), a self-shielding, mobile linear accelerator. Gross Tumor Volume (GTV) included the gross tumor based on CT and MRI imaging, if available. The Clinical Target Volume (CTV) was defined as GTV plus surrounding areas at risk for containing microscopic disease. The CTV included the GTV aiming at a margin of $1-4 \mathrm{~cm}$ if feasible, depending on the location and anatomy. The CTV margins were smaller if the GTV was adjacent to the critical normal organs like small bowel. For extremity desmoids one third of the skin circumference was spared in order to reduce the risk of chronic lymph edema. The PTV margins depend on localization and technique, for cranial localization a $3 \mathrm{~mm}$ margin was added to the CTV, extracranial $5 \mathrm{~mm}$ in most cases, for particles 7 $\mathrm{mm}$ in beam direction and $5 \mathrm{~mm}$ in the other directions. Proton therapy was preferred when technically possible and covered by patient's insurance $(N=15,34 \%)$. Carbon ion radiotherapy was used in one case of re-irradiation. Photon radiotherapy was performed in the remaining cases $(N=$ $28,66 \%)$. The median prescribed dose of the percutaneous radiotherapy was $54 \mathrm{~Gy} / \mathrm{Gy}$ (RBE) (range 39.6-66, IQR 50-60). The median applied dose was 54 Gy/ Gy (RBE) (range 30-66, IQR 50-60). Four patients had a preliminary termination of treatment, three for reasons other than radiotherapy. Four patients hat an IOERT boost with the current treatment (12 Gy). The median planning target volume was $967 \mathrm{ml}$ (range 84-4364 ml, IQR 447-1988).

\section{Statistical analysis}

Follow-up time was calculated from the beginning of treatment until the last presentation. Local progression free survival (LPFS) was defined as the time from beginning of treatment until progression of the target lesion. 
Table 1 Patient characteristics

\begin{tabular}{|c|c|c|}
\hline & $n$ & Percent \\
\hline Number of patients & 40 & \\
\hline Number of treatments & 44 & \\
\hline \multicolumn{3}{|l|}{ Gender } \\
\hline Female & 25 & 62.5 \\
\hline Male & 15 & 37.5 \\
\hline \multicolumn{3}{|l|}{ Age at diagnosis } \\
\hline Median (range) & 39 & $1-78$ \\
\hline \multicolumn{3}{|l|}{ Age at radiotherapy } \\
\hline Median (range) & 41 & $8-78$ \\
\hline \multicolumn{3}{|l|}{ Multifokal/ unifokal at first RT } \\
\hline Unifokal & 30 & 75 \\
\hline Multifokal & 10 & 25 \\
\hline \multicolumn{3}{|c|}{ Localization of treated tumor at first treatment } \\
\hline Abdominopelvic cavity & 11 & 27.5 \\
\hline Thoracic wall & 9 & 22.5 \\
\hline Extremity & 9 & 22.5 \\
\hline Abdominal wall & 4 & 10 \\
\hline Trunk (paravertebral) & 4 & 10 \\
\hline Head and neck & 3 & 7.5 \\
\hline \multicolumn{3}{|c|}{ Familial adenomatous polyposis (FAP) } \\
\hline No & 31 & 77.5 \\
\hline Yes & 9 & 22.5 \\
\hline \multicolumn{3}{|c|}{ Previous surgery in area of primary tumor } \\
\hline No (all/ thereof FAP patients) & $35 / 4$ & $87.5 / 10$ \\
\hline Yes (all/ thereof FAP patients) & $5 / 5$ & $12.5 / 12.5$ \\
\hline \multicolumn{3}{|c|}{ Histologically confirmed desmoid tumor } \\
\hline Yes (all/ thereof FAP patients) & $35 / 4$ & $87.5 / 10$ \\
\hline No (all/ thereof FAP patients) & $5 / 5$ & $12.5 / 12.5$ \\
\hline \multicolumn{3}{|l|}{ Pregnant at radiotherapy } \\
\hline Yes & 1 & 2.5 \\
\hline
\end{tabular}

PFS was defined as the time from beginning of treatment until progression of the target lesion, other lesions, new lesions or death. Survival analysis war performed from beginning of treatment until the first progression $(n=$ 40), the second treatment was not considered. Survival curves were calculated by the Kaplan-Meier method and compared by the log-rank test. Variables with a $p$-value of less than 0.05 were considered significant. Response of the target lesion was defined according to the Response Evaluation Criteria in Solid Tumors (RECIST 1.1) and evaluated at the end of the follow-up interval on the last available MRI or CT. Toxicity was assessed by Common Terminology Criteria for Adverse Events (CTCAE) v. 5.0, transferred to this version when previously graded with former versions, when grading was not available, the toxicity was stated as present.
Table 2 Radiotherapy timepoint and concept, previous treatment characteristics

\begin{tabular}{|c|c|c|}
\hline & $\mathrm{n}$ & Percent \\
\hline Number of treatments & 44 & \\
\hline \multicolumn{3}{|l|}{ Treatment timepoint } \\
\hline Recurrence/ progression & 33 & 75 \\
\hline At first diagnosis & 11 & 25 \\
\hline \multicolumn{3}{|l|}{ Treatment concept } \\
\hline Definitive RT & 27 & 61.4 \\
\hline Direct post-operative RT & 17 & 38.6 \\
\hline -Microscopically incomplete resection (R1) & 8 & 18.2 \\
\hline -Macroscopically incomplete resection (R2) & 5 & 11.4 \\
\hline -Unknown (Rx) & 4 & 9.1 \\
\hline \multicolumn{3}{|l|}{ Foregoing surgery of the treated desmoid tumor } \\
\hline No & 14 & 31.8 \\
\hline 1 resection & 18 & 40.9 \\
\hline 2 resections & 6 & 13.6 \\
\hline$>2$ resections & 6 & 13.6 \\
\hline \multicolumn{3}{|c|}{ Macroscopic tumor before RT on planning computed tomography } \\
\hline Yes & 31 & 70.5 \\
\hline No & 13 & 29.5 \\
\hline \multicolumn{3}{|l|}{ Previous radiotherapy } \\
\hline Percutaneous RT of desmoid tumor & 7 & 16.9 \\
\hline Overlap of high dose area & 5 & 11 \\
\hline \multicolumn{3}{|l|}{ Previous medical treatment } \\
\hline No & 26 & 59.1 \\
\hline Yes & 18 & 40.9 \\
\hline
\end{tabular}

\section{Results}

Survival analysis

The median follow-up time was 32 months [range 1153 months]. The local PFS was 79,4 and $63.8 \%$ at 3 and 5 years, respectively. The PFS from desmoid tumor of any location was 72.3 and $58.4 \%$ at 3 and 5 years, respectively. All but one patient were alive during the follow-up interval, resulting in an overall survival probability of $97.4 \%$ at 3 and 5 years (Fig. 2). In univariate analysis unifocal localization of the desmoid tumor was associated with superior PFS and age below the median of $41 \mathrm{y}$ with inferior PFS $(p=0.013$ and $p=0.002$, respectively, Supplemented Table 3). PFS did not differ significantly between patients with and without FAP, between patients with definitive vs. directs postoperative treatment as well as between the treatment at the time of progression or the time of the first diagnosis.

\section{Response analysis}

Follow-up imaging was available for 43 of 44 treatments, as one patient died soon after the end of radiotherapy. Of 31 patients with macroscopic tumor at beginning of 


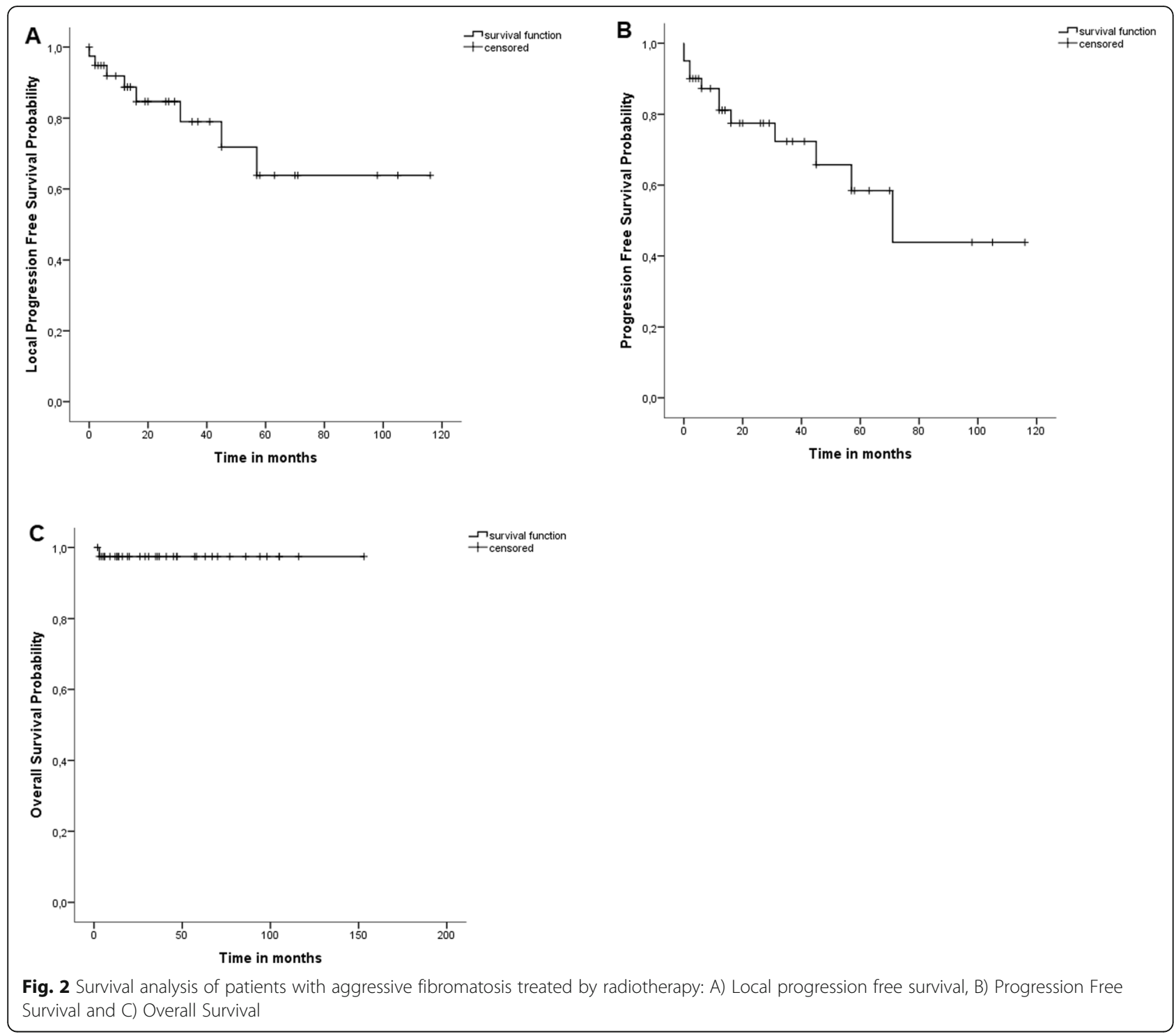

treatment 12 showed a partial remission of the tumor size $(38.7 \%)$ and four a complete remission (12.9\%). Stable disease was observed in eight cases $(25.8 \%)$ of this subcohort and progressive disease in six (19.4\%). A lower portion of treatments $(N=13,29.5 \%)$ was performed as postoperative radiotherapy after incomplete resection and had no/ unsure (=differentiation between possible residual tumor and postoperative surgical changes was not exactly feasible) macroscopic tumor on planning computed tomography, those patients showed complete remission in 10 (77\%) cases and progressive disease in three (23\%) cases (Table 4 ).

Recurrence occurred in 13 treatments. The pattern of recurrence was recurrence at the PTV margin $(N=5$, $38.4 \%)$, recurrence within the PTV $(N=4,30.8 \%)$ and outside the PTV $(N=2,15.4 \%)$ or in different anatomical region $(N=2,15.4 \%$, Table 4$)$.

\section{Toxicity}

Late toxicity depended on the localization, patients with extraabdominal desmoids mostly suffered from lymphedema $(N=8)$, fibrosis $(N=4)$, decreased range of joint motion $(N=7)$ or sensory disturbance/paresis $(N=4)$ (Supplemented Table 2).

Patients with FAP and intraabominal desmoid tumors had a tendency for severe complications. A 44-year-old patient developed chylous ascites after radiotherapy for intraabdominal multilocalized desmoid tumors. He developed peritonitis and required opening of an abscess after paracentesis. Several times the patient developed sepsis which was contributed to cholangitis. In the location of the previous desmoid he developed an interenteric retention due to fistula. After five consecutive operations for persisting multiple fistula and perforations a jejunostomy was performed and the 
Table $\mathbf{3}$ Treatment characteristics

\begin{tabular}{|c|c|c|c|}
\hline \multirow{2}{*}{\multicolumn{4}{|c|}{ RT Technique }} \\
\hline & & & \\
\hline \multicolumn{2}{|l|}{ Photon } & 28 & 66 \\
\hline \multicolumn{2}{|l|}{ 3-D conformal RT } & 8 & 18.2 \\
\hline \multicolumn{2}{|l|}{ IMRT } & 20 & 45.5 \\
\hline \multicolumn{2}{|l|}{ Particle } & 16 & 36.4 \\
\hline \multicolumn{2}{|l|}{ Protons } & 15 & 34 \\
\hline \multicolumn{2}{|l|}{ Carbon ion } & 1 & 2.3 \\
\hline \multicolumn{4}{|c|}{ Prescribed total dose (Gy/ Gy (RBE)) } \\
\hline \multicolumn{2}{|l|}{ Median (Range, IQR) } & 54 & $39.6-66,50-60$ \\
\hline \multicolumn{2}{|l|}{$<50$} & 5 & 11 \\
\hline \multicolumn{2}{|l|}{$\geq 50$} & 24 & 55 \\
\hline \multicolumn{2}{|l|}{$\geq 60$} & 15 & 34 \\
\hline \multicolumn{4}{|l|}{ Single dose } \\
\hline Proton & 1.8 Gy/ Gy (RBE) & 14 & 31.8 \\
\hline Photon & 2 Gy/ Gy (RBE) & 29 & 65.9 \\
\hline Carbon ion & 3 Gy (RBE) & 1 & 2.3 \\
\hline \multicolumn{2}{|c|}{ Volume reduction (percutaneous boost) } & 4 & 9.1 \\
\hline \multicolumn{4}{|c|}{ Intraoperative electron radiation therapy (IOERT) boost } \\
\hline & 12 Gy & 4 & 8.4 \\
\hline \multicolumn{4}{|c|}{ Planning target volume (ml) } \\
\hline & Median (range, IQR) & 967.06 & $(84-4364,447-1988)$ \\
\hline & Missing & 1 & 2.3 \\
\hline \multicolumn{2}{|c|}{ Premature discontinuation of RT } & 4 & 9.1 \\
\hline
\end{tabular}

Table 4 Outcome characteristics

\begin{tabular}{|c|c|c|c|}
\hline & & $\mathrm{n}=44$ & Percent \\
\hline \multicolumn{2}{|c|}{ Response of target lesion with macroscopic tumor before RT } & 31 & 70.5 \\
\hline & Complete Remission & 4 & 12.9 \\
\hline & Partial remission & 12 & 38.7 \\
\hline & Stable disease & 8 & 25.8 \\
\hline & Progressive disease & 6 & 19.4 \\
\hline & Missing & 1 & 3.2 \\
\hline \multicolumn{2}{|c|}{ Response of target lesion without macroscopic tumor before RT } & 13 & 29.5 \\
\hline & Complete remission & 10 & 77 \\
\hline & Progressive disease & 3 & 23 \\
\hline \multirow[t]{5}{*}{ Patterns of recurrence } & & 13 & 29.5 \\
\hline & At the PTV Margin & 5 & 38.4 \\
\hline & Within the PTV & 4 & 30.8 \\
\hline & Same anatomical region, outside the PTV & 2 & 15.4 \\
\hline & Different anatomical region & 2 & 15.4 \\
\hline
\end{tabular}


patient received a total parenteral nutrition (Supplemented Table 1).

One female patient (age 33 at the time of treatment) developed an abscess and a chronic inflammation in the location of the treated desmoid tumor which formed a fistula and a persisting ulcus to the neighboring ileo-anal pouch.

The treatment of one FAP patient with a central superinfected desmoid tumor was discontinued at 34.2 Gy before reaching the prescribed dose due to further progression of the abscess and progression in size of the desmoid tumor.

One patient with FAP, who has been treated for abdominopelvic aggressive fibromatosis, developed rectal bleeding and required cardiopulmonary resuscitation on the last day of radiotherapy. Furthermore, he developed a fistula between the treated desmoid and the adjacent bowel. He received drainage, however sepsis persisted. He died 2 months after the end of radiotherapy in palliative care.

Besides that, there were three further discontinuations, two for reasons other than the desmoid or its treatment and one was the case of a female patient who was pregnant at diagnosis of a gigantic cervico-thoracic desmoid tumor which presented with immense neuropathic pain due to damage of the cervical plexus, stricture of the upper respiratory tract and difficulties in breathing and swallowing. The patient was treated beginning in the 28th week of pregnancy with protons, however the tumor progressed rapidly. Consequently, the treatment was discontinued and Caesarean section was performed. Under the treatment with methotrexate and vinorelbine the tumor regressed significantly after birth (Fig. $1 \mathrm{C}$ and D).

\section{Discussion}

Desmoid tumors are rare and large or randomized series are not available up to date, thus there is no evidencebased approach. Characteristically the course of the disease is variable and difficult to predict.

In recent years immediate surgery especially for asymptomatic or paucisymptomatic desmoid tumors has been increasingly restricted 7 [2], as it has been observed that watchful waiting can achieve PFS rates of $50 \%$ in 5 years. A conservative, nonaggressive policy has been based on the ability of extra abdominal desmoid tumors to spontaneously stabilize [10-12]. Even spontaneous regressions are possible in $20 \%$ of cases, thus the tendency to follow with observation only is growing [5].

When local therapy is necessary surgery or radiotherapy or both form the options. Preservation of function is a priority; mutilating surgery has to be avoided and radiotherapy can be beneficial in selected cases esp. in the treatment of head and neck or intrathoracic desmoids [2]. A comparative review of 22 articles published by Nuyttens et al. showed that local control after treatment with a combination of surgery and radiotherapy was significantly improved compared to surgery alone $(75 \%$ vs $61 \%)$, this applied to surgically positive and negative margins as well as primary and recurrent tumors. This study (like most) excluded articles with FAP, children, abdominal localization and head and neck because here response to treatment can be less or more favorable [13]. In our cohort younger age and multilocal localization was associated with worse survival, FAP showed however no significant difference. A second more recent meta-analysis of in total 1295 patients showed that the risk of local recurrence was twofold higher for those with positive resection margins. Adjuvant RT was only beneficial in case of incomplete resection for primary but particularly for recurrent tumors [14]. On the other hand, it has been shown for desmoid tumors of the abdominal wall that of 41 patients only one developed a recurrence within 97 months after incomplete (R1) resection [4]. To date the benefit for postoperative radiotherapy is not conclusively clarified. In cases of recurrence after previous surgery and when local control is crucial, postoperative radiotherapy can be considered. It is worth noting that a significant portion our cohort $(N=17)$ was presented after incomplete resection and 12 patients were presented with recurrent disease after two or more resections. Previously no significant differences in terms of local control were reported for radiotherapy alone compared to the combination of radiotherapy and surgery [15]. Local control after radiation therapy was shown to be better for patients with fewer than two operations versus more than three [16]. Additionally, recurrence seems to be a significant unfavorable independent risk factor for further recurrence [17]. Taking all this into account, one might consider definitive radiotherapy when the risk of incomplete resection is high in order to avoid the need of two subsequent treatment modalities and the combination of the according toxicities.

A phase II pilot study $(n=44)$ for patients of inoperable progressive desmoid tumors of the trunk and extremities established a dose of 56 Gy in 28 fractions. The response rates of the cohort were similar to our subcohort of patients with macroscopic tumors at beginning of radiotherapy regarding partial remission $(36.4 \%)$ and complete remission (13.6\%) [18]. However, the rate of stable disease was higher compared (25.8\% vs $40.9 \%)$ und the rate of progressive disease lower (19.4\% vs. $6.8 \%$ ). Compared to many tumors of mesenchymal origin this reduction in size is striking, as in many tumors with low proliferation indices (e.g. low-grade chondrosarcoma, liposarcoma) a stationary size with assumed biological inactivation characterize the treatment 
response of the tumor. Similar local control rates of $79 \%$ after a median follow-up of 44 months were reported by the German Cooperative Group on Radiotherapy for Benign Diseases (GCG-BD) [19].

Proton therapy has a superior dose distribution compared to percutaneous photon therapy and allows a better sparing of surrounding organs at risk with potential in reducing acute and long-term toxicity. Retrospective data on a cohort of 115 desmoid patients treated between 1965 and 2005 showed a rate of radiation related complications of $17 \%$ with a median follow-up of 10.1 years [15], the potential of particle therapy to reduce this rates has to be investigated further in future. The main concern about radiotherapy is the risk of secondary malignancy e.g. radiation induced sarcoma in a young population with substantial chances of long term survival. The medium and low dose of protons to surrounding tissue is significantly lower on dosimetric comparison and thus the risk of secondary malignancy is reduced [20]. In clinical cohorts of adults as well as pediatric patients low rates of secondary malignancies have been reported for the treatment with protons [21, $22]$. In a large case matched comparison, the rates were 5.2 and $7.5 \%$ for the proton and photon cohort, respectively [21]. Whenever available and technically possible we thus strongly recommend the use of proton therapy, in analogy to the recommendations for pediatric patients. In case of re-irradiation we tend to rely on carbon-ions as the lateral dose gradients are even stepper than those of protons and thus treatment related morbidity is reduced [23]. Aside from the case of reirradiation no recommendations can be made regarding carbon ion therapy due to the very limited experience.

Historically we aimed at prescription doses of approximately 60 Gy in parallel to other tumors of mesenchymal origin. However, close location to sensitive organs at risk made a reduction of the dose necessary and we achieved in this cohort a median dose of $54 \mathrm{~Gy} / \mathrm{Gy}$ (RBE) with the here presented promising results. The latest consensus guidelines of the Desmoid Working Group suggest a "moderate dose radiotherapy" in those cases when active therapy is necessary but the risk of surgery-associated long-term morbidity is high [6]. In parallel, the previous European Consensus Guideline recommended 56 Gy in 28 fractions, based on the result of the aforementioned phase II study [2]. It is worth noting that a dose of 50 Gy/ Gy (RBE) or lower was associated with impaired survival on univariate analysis in our cohort $(p=0.014)$, thus our data support a prescription dose of $\sim 56 \mathrm{~Gy} / \mathrm{Gy}$ (RBE).

The risk of progression during pregnancy is high, however a desmoid tumor is not a contradiction for future pregnancies. Although it is described that less than 50\% of patients require treatment [24], this was not the case with the patient described here due to the gigantic size and rapid progression of the tumor compressing the upper airways and esophagus. We included this seldom case in our analysis, to give an impression on the level of local aggressiveness a desmoid tumor can develop.

FAP- associated desmoid type fibromatosis has to be differed from the sporadic tumors. Commonly they are larger, multiple and often intra-abdominal. The risk of recurrence is furthermore higher (44\% vs. 25\%) and among the FAP-patients desmoids constitute a significant cause of death [25]. The treatment is even more challenging. Tumor related complications are common and include intestinal obstruction, perforation or ischemia as well as urethral obstruction [26]. We observed severe acute and late toxicity with radiotherapy for FAPassociated desmoid tumors in our series. Necrosis, abscess formation [27, 28] and fistula has been described previously for desmoid patients that have not been treated by radiotherapy. Our data show that radiotherapy although highly effective in the reduction of size cannot prevent and might even promote the formation of these complications. Based on this experience we conclude that RT in patients with FAP-associated desmoid tumors should be only initiated when other options have been exploited.

\section{Conclusion}

The limitations of the study lie in the retrospective character. We deliberately did not exclude patients with head and neck desmoids, FAP-associated desmoids or juvenile desmoids, which can influence the estimated local progression free survival [25, 29, 30], however provides more comprehensive information regarding treatment related morbidity. Aside of FAP-associated desmoids, RT was safe and feasible and contributed to local control with good chances of even reduction in tumor size. Limited evidence hinders the establishment of unequivocal guidelines; thus, treatment decisions have to be obtained interdisciplinary and a personalized management considering the varying locations and the possibly associated tumor-related and treatment-related complications has to be established.

\section{Supplementary information}

Supplementary information accompanies this paper at https://doi.org/10. 1186/s13014-020-01565-9.

\section{Additional file 1}

\section{Abbreviations}

CTCAE: Common Terminology Criteria for Adverse Events; CTV: Clinical target volume; GTV: Gross tumor volume; FAP: Familial adenomatous polyposis; GCG-BD: German Cooperative Group on Radiotherapy for Benign Diseases: HIT: Heidelberg Ion-Beam Therapy Center; IMRT: Intensity modulated radiotherapy; LPFS: Local progression free survival; PFS: Progression free survival; PTV: planning target volume; OS: Overall survival; RBE: Relative 
biological effectiveness; RT: radiotherapy; VMAT: Volumetric modulated arc therapy

\section{Acknowledgments}

Katharina Seidensaal is funded by the Rahel-Goitein-Straus program.

\section{Authors' contributions}

$\mathrm{KS}, \mathrm{JD}$ and MU contributed conception and design of the study; SH, FW, TW, $\mathrm{GM}, \mathrm{BL}, \mathrm{MS}, \mathrm{SF} \mathrm{GE}$ and $\mathrm{KH}$ provided the data; $\mathrm{KS}, \mathrm{SH}, \mathrm{FW}$, and $\mathrm{MU}$ analyzed the data; KS, FW, MU, KH and JD wrote the manuscript; All authors contributed to manuscript revision, read and approved the submitted version.

\section{Funding}

Not applicable.

\section{Availability of data and materials}

The datasets used and/or analyzed during the current study are available from the corresponding author on reasonable request.

\section{Ethics approval and consent to participate}

This study was performed following institutional guidelines and the Declaration of Helsinki of 1975 in its most recent version. This study was approved by the Independent local Ethics Committee Heidelberg (Ref. Nr. S119/2019)

\section{Consent for publication}

Not applicable.

\section{Competing interests}

The research was conducted in the absence of any commercial or financial relationships that could be construed as a potential conflict of interest.

\begin{abstract}
Author details
'Department of Radiation Oncology, Heidelberg University Hospital, Im Neuenheimer Feld 400, 69120 Heidelberg, Germany. ${ }^{2}$ Heidelberg Institute of Radiation Oncology (HIRO), Heidelberg, Germany. ${ }^{3}$ National Center for Tumor diseases (NCT), Heidelberg, Germany. ${ }^{4}$ Heidelberg lon-Beam Therapy Center $(H I T)$, Department of Radiation Oncology, Heidelberg University Hospital, Heidelberg, Germany. ${ }^{5}$ Clinical Cooperation Unit Radiation Oncology, German Cancer Research Center (DKFZ), Heidelberg, Germany. ${ }^{6}$ Institute of Pathology, University of Heidelberg, Heidelberg, Germany. ${ }^{7}$ Center for Orthopedics, Trauma Surgery and Paraplegiology, University of Heidelberg, Heidelberg, Germany. ${ }^{8}$ Department of General, Visceral and Transplantation Surgery, University Hospital Heidelberg, Heidelberg, Germany. ${ }^{9}$ Department of Translational Medical Oncology, National Center for Tumor Diseases Heidelberg and German Cancer Research Center, Heidelberg, Germany. ${ }^{10}$ Department of Hematology, Oncology and Rheumatology, Heidelberg University, Heidelberg, Germany. ${ }^{11}$ German Cancer Consortium (DKTK), partner site Heidelberg, Heidelberg, Germany.
\end{abstract}

\section{Received: 11 February 2020 Accepted: 8 May 2020}

Published online: 05 June 2020

\section{References}

1. Kasper B, Strobel P, Hohenberger P. Desmoid tumors: clinical features and treatment options for advanced disease. Oncologist. 2011;16(5):682-93.

2. Kasper B, et al. An update on the management of sporadic desmoid-type fibromatosis: a European consensus initiative between sarcoma PAtients EuroNet (SPAEN) and European Organization for Research and Treatment of Cancer (EORTC)/soft tissue and bone sarcoma group (STBSG). Ann Oncol. 2017:28(10):2399-408

3. Escobar C, et al. Update on desmoid tumors. Ann Oncol. 2012;23(3):562-9.

4. Bonvalot S, et al. Spontaneous regression of primary abdominal wall desmoid tumors: more common than previously thought. Ann Surg Oncol. 2013;20(13):4096-102

5. Colombo C, et al. Sporadic extra abdominal wall desmoid-type fibromatosis: surgical resection can be safely limited to a minority of patients. Eur Cancer. 2015;51(2):186-92.
6. Alman B, et al. The management of desmoid tumours: a joint global consensus-based guideline approach for adult and paediatric patients. Eur J Cancer. 2020;127:96-107.

7. Biermann JS. Desmoid tumors. Curr Treat Options in Oncol. 2000;1(3):262-6.

8. Combs SE, et al. Heidelberg ion therapy center (HIT): initial clinical experience in the first 80 patients. Acta Oncol. 2010;49(7):1132-40.

9. Rieken $\mathrm{S}$, et al. Assessment of early toxicity and response in patients treated with proton and carbon ion therapy at the Heidelberg ion therapy center using the raster scanning technique. Int J Radiat Oncol Biol Phys. 2011;81(5): e793-801.

10. Lewis JJ, et al. The enigma of desmoid tumors. Ann Surg. 1999;229(6):86672 discussion 872-3.

11. Bonvalot $\mathrm{S}$, et al. Extra-abdominal primary fibromatosis: aggressive management could be avoided in a subgroup of patients. Eur J Surg Oncol. 2008;34(4):462-8.

12. Fiore $\mathrm{M}$, et al. Desmoid-type fibromatosis: a front-line conservative approach to select patients for surgical treatment. Ann Surg Oncol. 2009;16(9):258793.

13. Nuyttens JJ, et al. Surgery versus radiation therapy for patients with aggressive fibromatosis or desmoid tumors. Cancer. 2000;88(7):1517-23.

14. Janssen $\mathrm{ML}$, et al. Meta-analysis of the influence of surgical margin and adjuvant radiotherapy on local recurrence after resection of sporadic desmoid-type fibromatosis. Br J Surg. 2017;104(4):347-57.

15. Guadagnolo BA, Zagars GK, Ballo MT. Long-Term Outcomes for Desmoid Tumors Treated With Radiation Therapy. Int J Radiation Oncol Biol Physics. 2008:71(2):441-7.

16. Sherman NE, et al. Desmoid tumors: a 20-year radiotherapy experience. Int $J$ Radiat Oncol Biol Phys. 1990;19(1):37-40.

17. Posner MC, et al. The desmoid tumor. Not a benign disease. Arch Surg. 1989;124(2):191-6.

18. Keus RB, et al. Results of a phase II pilot study of moderate dose radiotherapy for inoperable desmoid-type fibromatosis--an EORTC STBSG and ROG study (EORTC 62991-22998). Ann Oncol. 2013:24(10):2672-6.

19. Kriz J, et al. Radiotherapy is effective for desmoid tumors (aggressive fibromatosis) - long-term results of a German multicenter study. Oncol Res Treat. 2014;37(5):255-60

20. Eaton $\mathrm{BR}$, et al. Secondary malignancy risk following proton radiation therapy. Front Oncol. 2015;5:261.

21. Chung CS, et al. Incidence of second malignancies among patients treated with proton versus photon radiation. Int J Radiat Oncol Biol Phys. 2013; 87(1):46-52

22. Yock TI, et al. Long-term toxic effects of proton radiotherapy for paediatric medulloblastoma: a phase 2 single-arm study. Lancet Oncol. 2016;17(3):28798

23. Seidensaal K, et al. Re-irradiation with protons or heavy ions with focus on head and neck, skull base and brain malignancies. Br J Radiol. 2020:93(1107).

24. Fiore $\mathrm{M}$, et al. Desmoid-type fibromatosis and pregnancy: a multiinstitutional analysis of recurrence and obstetric risk. Ann Surg. 2014;259(5): 973-8.

25. Koskenvuo L, Ristimaki A, Lepisto A. Comparison of sporadic and FAPassociated desmoid-type fibromatoses. J Surg Oncol. 2017;116(6):716-21.

26. Santos $\mathrm{M}$, et al. Desmoid Tumours in Familial Adenomatous Polyposis: Review of 17 Patients from a Portuguese Tertiary Center. J Clin Diagnostic Res. 2016;10(10):PC01-5

27. Alemanno $G$, et al. A multidisciplinary approach to desmoid tumors. When intra-abdominal fibromatosis degenerates into an abscess, which is the right treatment? Int J Surg Case Rep. 2013:4(9):757-60.

28. Maldjian C, et al. Abscess formation in desmoid tumors of Gardner's syndrome and percutaneous drainage: a report of three cases. Cardiovasc Intervent Radiol. 1995;18(3):168-71.

29. de Bree $\mathrm{E}$, et al. Desmoid tumors of the head and neck: a therapeutic challenge. Head Neck. 2014;36(10):1517-26.

30. Honeyman JN, Quaglia MPL. Desmoid tumors in the pediatric population. Cancers. 2012:4(1):295-306.

\section{Publisher's Note}

Springer Nature remains neutral with regard to jurisdictional claims in published maps and institutional affiliations. 